Copyright (C) 2008 IEEE.

Reprinted from the IEEE Radar Conference: pp.1514-1519

This material is posted here with permission of the IEEE. Such permission of the IEEE does not in any way imply IEEE endorsement of any of the University of Adelaide's products or services. Internal or personal use of this material is permitted. However, permission to reprint/republish this material for advertising or promotional purposes or for creating new collective works for resale or redistribution must be obtained from the IEEE by writing to pubs-permissions@ieee.org.

By choosing to view this document, you agree to all provisions of the copyright laws protecting it. 


\title{
A PRACTICAL BISTATIC PASSIVE RADAR SYSTEM FOR USE WITH DAB AND DRM ILLUMINATORS
}

\author{
C.J.Coleman ${ }^{1}$, R.A.Watson ${ }^{2}$, and H.Yardley ${ }^{l}$ \\ ${ }^{1}$ School of Electrical and Electronic Engineering, The University of Adelaide \\ Adelaide, SA 5005, Australia \\ email: ccoleman@.eleceng.adelaide.edu.au, hyardley@eleceng.adelaide.edu.au \\ ${ }^{2}$ Department of Electronic and Electrical Engineering, The University of Bath \\ Bath, BA2 7AY, UK \\ email: R.J.Watson@bath.ac.uk
}

Keywords: Passive Bistatic Radar, Digital Audio Broadcasting, Digital Radio Mondiale.

\begin{abstract}
Digital broadcasts provide powerful illuminators of opportunity with waveforms that produce excellent radar ambiguity functions. An experimental program to investigate the possibility of such broadcasts for target tracking radar is described. In addition, the atmospheric monitoring possibilities for such broadcasts are discussed.

\section{INTRODUCTION}

The basic idea of passive bistatic radar (PBR) is one of measuring radar returns from targets that have been illuminated by non cooperative transmitters. Illuminations can be provided by completely unrelated radio systems and so radar observations can be clandestine. The notion of using transmitters of opportunity has been around for some time (see [1] to [8]). In particular, passive radar based on commercial broadcasts is well developed in the form of Silent Senty [2]. A large number of papers have been written on the subject and the reader should consult Griffiths [1] for a summary of the relevant literature. What has changed in recent years is the nature of the available illuminators. In particular, the move towards digital commercial broadcasting has provided a unique opportunity for PBR sine the pseudo random nature of the digital modulation can give an almost ideal radar ambiguity function. Poullin [8] has considered the use of digital broadcasts for PBR, but his results are inconclusive. We show, in this paper, that digital broadcasts can be used for the basis of a successful PBR.
\end{abstract}

For a standard analogue FM transmission, a typical ambiguity function is shown in fig. 1. It will be noted that the function is far from ideal with a large spread in both Doppler and range. Furthermore, the example is for rock music modulation which has arguably the best performance for radar purposes. Up to now, the poor resolution obtained with analogue transmissions has been one of the major stumbling blocks to passive radar based on commercial broadcasts. We would like to use such transmissions because of their power, but we also need good resolution. With digital broadcasting we have the power together with the resolution. Fig. 2 shows the ambiguity function for a typical digital audio broadcast (DAB) taken from the UK system. It will be noted that the function has a close to ideal thumbtack nature, a result of the pseudo random nature of the modulation. DAB systems run at frequencies of just over $200 \mathrm{MHz}$ with a bandwidth of just over $1.5 \mathrm{MHz}$ and so can form the basis of PBR with good range resolution. Each transmitter has an output power of the order of $10 \mathrm{~kW}$ ERP and, in the UK, they are arranged as a network that transmits virtually identical signals. Consequently, such broadcasts have the potential to provide a radar system that covers a large area.

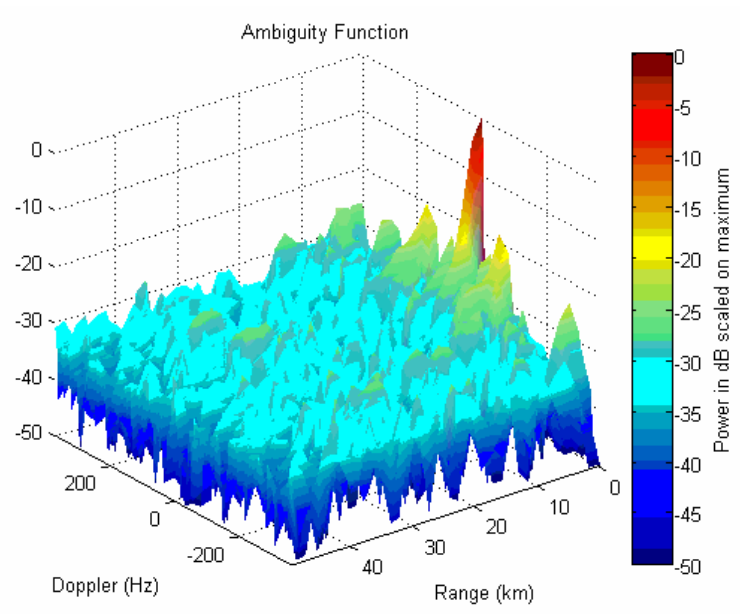

Figure 1 - Typical off air FM ambiguity function

Another fast growing system of digital broadcasting is provided by Digital Radio Mondiale (DRM). DRM broadcasts have a much narrower bandwidth than DAB (typically $10 \mathrm{kHz}$ ) and tend to transmit on much lower frequencies (short and medium waves). Fig. 3 shows a typical off air ambiguity function for a DRM transmission. This has, once again, a thumbtack nature, but the potential range resolution 
is relatively poor due to the narrow bandwidth of the DRM signal. Furthermore, there are additional peaks in this off air signal that are caused by the multiple propagation modes that are always present in ionospheric propagation. Nevertheless, the large powers emitted by some DRM broadcasters open up the possibility of its use in radar. There is even the possibility of PBR over the horizon radar (OTHR), particularly in surface wave mode.

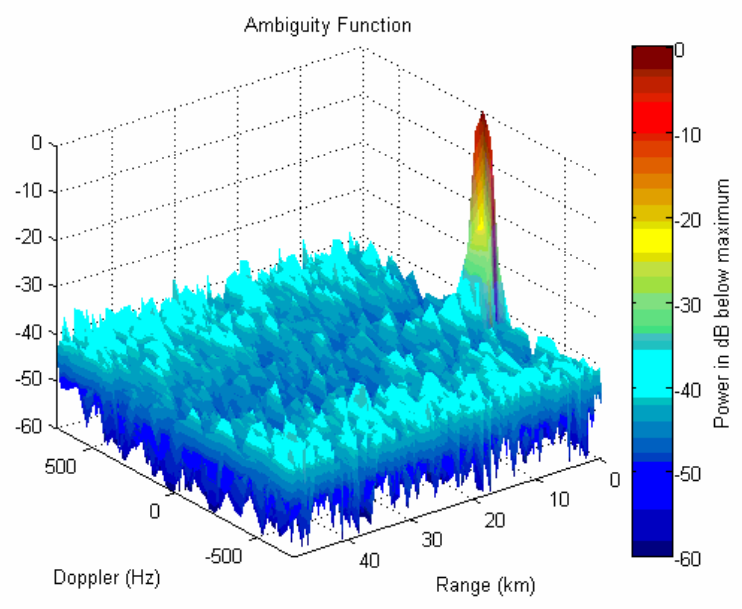

Figure 2 - Typical DAB ambiguity function

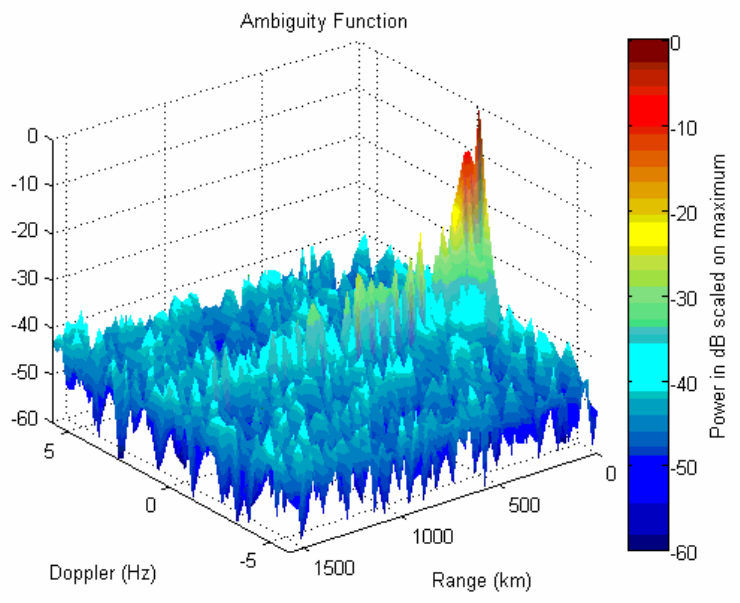

Figure 3 - Typical off air DRM ambiguity function

Digital Video Broadcasting (DVB) is a high power system that also offers great potential for PBR, but it has a much more complex signal structure that requires far more complex signal processing. There are several other digital transmissions of opportunity, but most are relatively weak (GPS for example) or erratic in transmissions (mobile telephone systems for example).

The University of Adelaide has developed a PBR test bed [9] which, for the past three years, has been located at the University of Bath in the UK. The location was chosen because of the rich environment of both DAB and DRM illuminators in its vicinity and the proximity of Bristol airport. In the current paper, we describe some of the observations using this test bed and discuss some of the problems encountered in implementing a PBR system based on digital radio broadcasts. In addition to more conventional aircraft tracking applications, we discuss the potential for the use of digital broadcasts in monitoring atmospheric effects. In section 2 of this paper we describe the test bed, in section 3 we detail some aircraft observations using DAB broadcasts and in section 4 we describe some current work on the use of digital broadcasts for atmospheric and ionospheric observations. In section 5 we discuss some of the problems that still need to be addressed and possible future directions for the work.

\section{RADAR TEST BED}

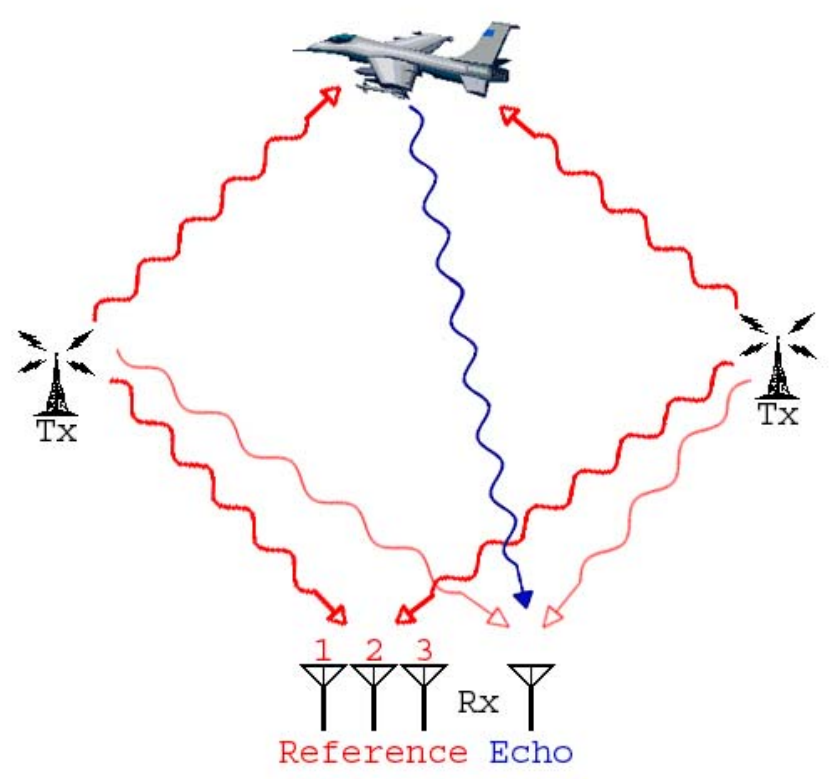

Figure 4 - Typical passive radar setup.

The radar test bed [9] consists of the ICS554B four channel digital receiver, a computer, three Yagi-Uda antennas, a fixed array of Yagi-Uda antennas, phasing networks and some antenna amplifiers. For radar experiments using DAB, the radar uses digital down conversion and so the system also includes a bank of bandpass filters centered on the chosen DAB frequencies. The array of Yagi-Uda antennas is pointed towards the target area and the other three antennas are pointed respectively towards the three most powerful DAB illuminators (see fig. 4). The DAB illuminators transmit on identical frequencies and have almost identical waveforms, the minute difference in waveform being an identifier in the transmitted signal. There are, however, delay differences between the illuminator signals due to their different locations. One of the main problems with the detection of targets is that, even though the target array is directed towards the targets, it can still have an appreciable response towards the illuminators. This effect is known as direct signal interference (DSI). Since the illuminator signals are massive in comparison to the target returns, there needs to be a 
way of reducing the effect on the target receiver. The most obvious method is to make target observations using opposite polarisation to that of the DAB transmissions (vertical polarisation). This considerably reduces the direct signal and is acceptable as most targets give appreciable returns in cross polarisation. Additionally, however, some form of illuminator cancellation is required. Signals from each of the three illuminator directed antennas are suitably delayed, and attenuated, before being combined with the output from the target antenna and the combination fed into the target channel of the digital receiver. The delays, and attenuation, are chosen so that the power of direct illuminations is minimized within this channel of the receiver. This ensures that there is maximum dynamic range within the digital receiver for target detection.

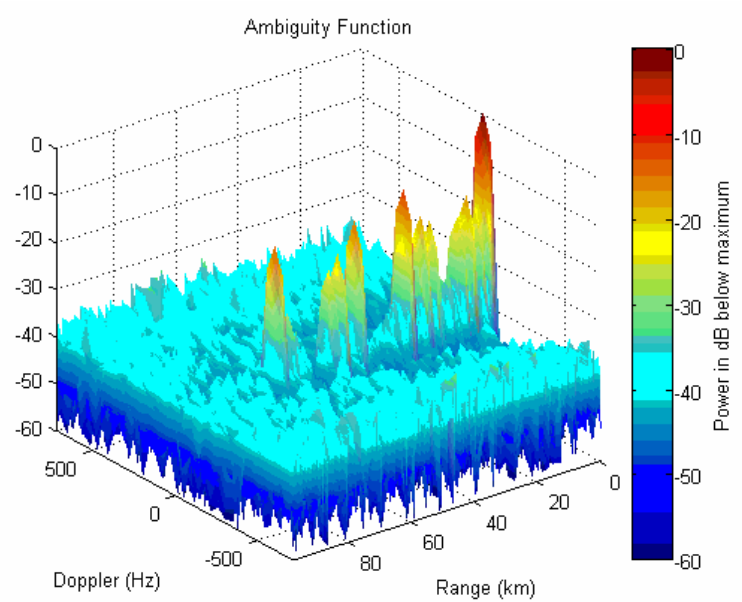

Figure 5 - Ambiguity for target channel before cancellation.

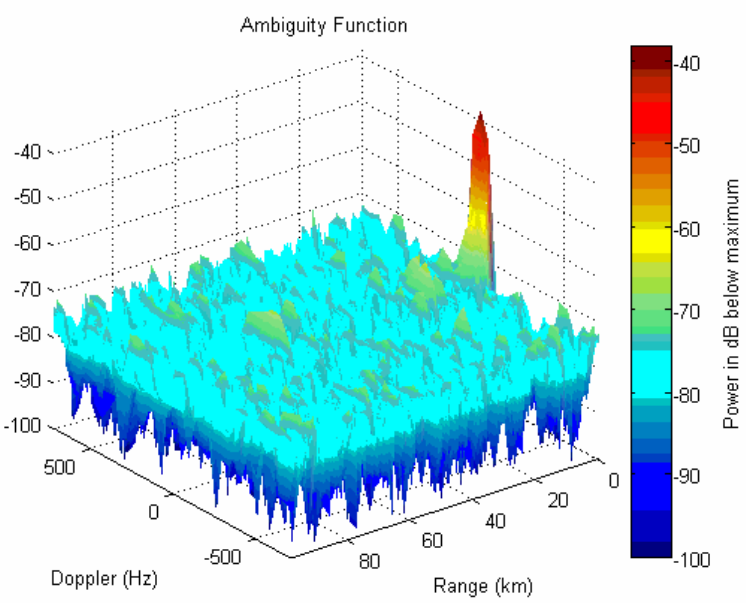

Figure 6-Ambiguity function after digital cancellation.

Even after this analogue cancellation, the illuminator signals can still be so strong as to cause the noise floor, after correlation, to be well above the level of the radar returns. Consequently, a second stage of cancellation is applied in the digital domain. Output from the three illuminator directed antennas is fed into the three remaining channels of the digital receiver and FIR filters applied to each receiver output.
The filters are chosen so that, when their outputs are combined, they form a 'best fit' to the output of the target channel. When subtracted from the target channel, this forms a signal with a majority of the direct illumination cancelled out. Fig. 5 shows an example of the ambiguity function of the target channel before the digital cancellation and fig. 6 shows the same channel after such cancellation. The effectiveness of the cancellation process can be seen from the large drop in noise floor (fig. 6 is scaled on the maximum in fig.6). The process requires filters that are long enough to take into account the relative delays between the illuminator signals and so can be very computationally expensive and difficult to achieve in real time. Furthermore, the filters are adaptive and need to be recalculated at each observation. This has lead to the development of specialized solution techniques for the equations that must be solved in order to find the filter coefficients. These techniques utilize a combination of the conjugate gradient method and fast Fourier transform techniques. This combination results in an algorithm that can keep up with the real time requirements of the radar (the full details will be described elsewhere).

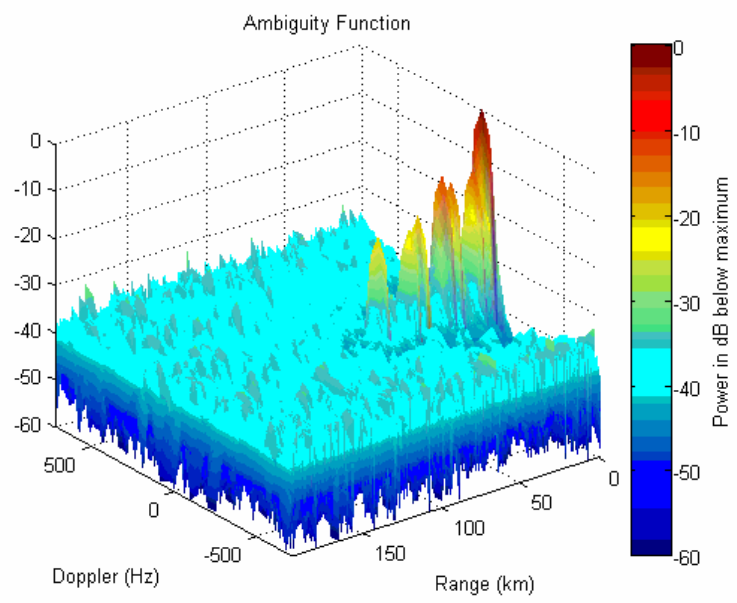

Figure 7 - Typical illuminator ambiguity before digital filtering.

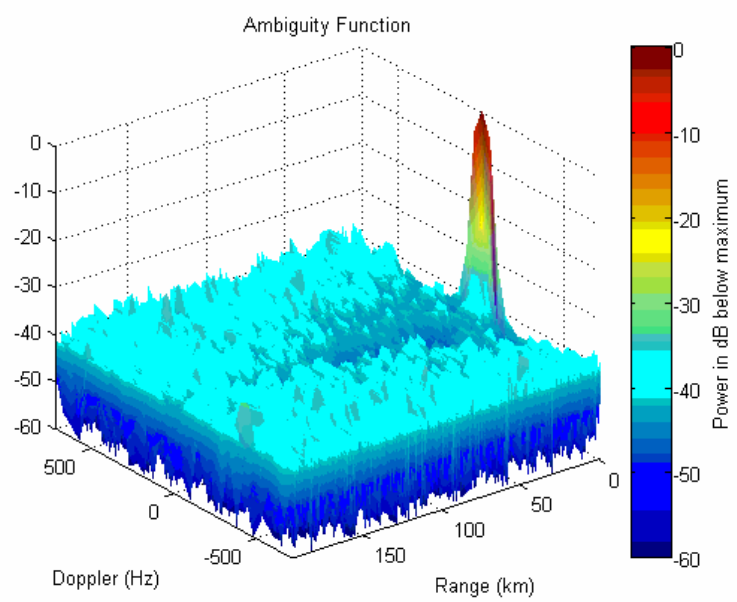

Figure 8 - Typical illuminator ambiguity after digital filtering. 
The detection of targets will also require a copy of the original transmitted signal for correlation purposes. Once again, the derivation of a suitable correlation signal poses a challenge in that antennas pointing towards a given illuminator will still have a response in the direction of the other illuminators. Consequently, the raw output from an illuminator channel is not the original transmitted signal, but will consist of a combination of the original signal at a variety of delays. Once again, the interfering signals can be removed by a FIR filter. The same solutions techniques that were developed for the target channel filter can also be applied and the algorithm can operate in real time. Fig. 7 shows the ambiguity function of a channel without the filtering and fig. 8 shows it with the filtering. It will be noted that the function shows that the filtering has removed most of the multiple copies to leave an estimate of the originally transmitted signal.

\section{AIRCRAFT OBSERVATIONS}

Once the direct illumination signals have been eliminated (or sufficiently reduced) in the target directed radar channel, and one of the illuminator channels cleaned to reveal the originally transmitted signal, the two can be correlated and a threshold applied to detect possible radar returns. Fig. 9 shows a typical result of such correlation after a threshold of $9 \mathrm{~dB}$ above the mean power level has been applied. The radar test bed was located at the University of Bath in the UK and the antennas pointed towards Bristol airport in order to observe plans traveling in and out. The range is in terms of the difference between the distance travelled by the direct signal (that from which the originally transmitted signal is derived) and that travelled by the signal reflected from the target. For this observation, the target (a Boeing 737 aircraft) is about $5 \mathrm{~km}$ from the radar and has a Doppler of about $100 \mathrm{~Hz}$. Figs. 10 shows an observation 20s later and fig. 11 shows the accumulated detections (i.e. tracks).

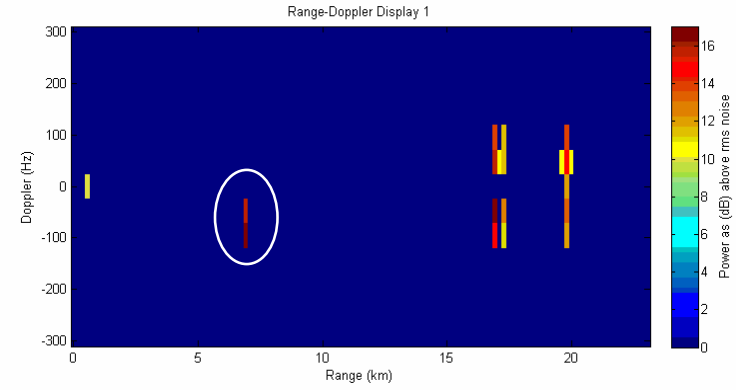

Figure 9 - Aircraft at relative range $7 \mathrm{~km}$ and Doppler $100 \mathrm{~Hz}$.

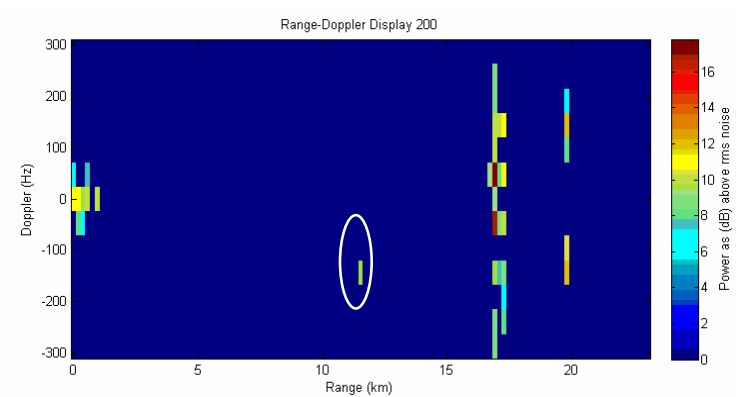

Figure 10 - Aircraft at relative range $12 \mathrm{~km}$ and Doppler $150 \mathrm{~Hz}$.
From the figures, it will be noted that there are also persistent returns around the ranges of 17 and $20 \mathrm{~km}$. These correspond to the ranges of the direct signals. Digital cancellation has removed nearly all the signal at zero Doppler and this has brought the noise floor down to a level where the target is clearly visible. This cancellation, however, has not removed that part of the direct signal that has been Doppler smeared and this could ultimately limit the sensitivity of the radar. The removal of such signals is an important area for further research if DAB bistatic radar is to achieve its full potential.

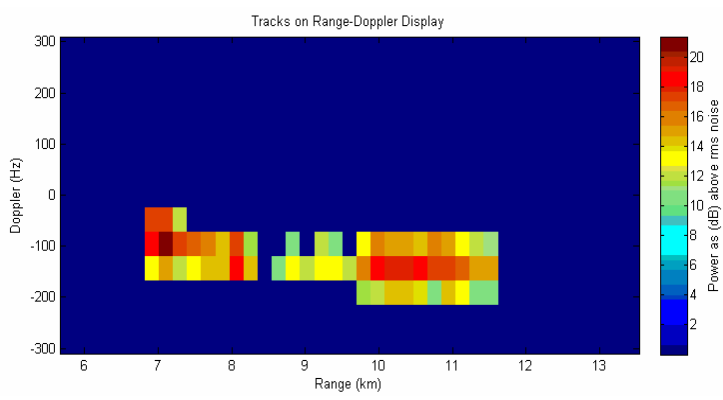

Figure 11 - Accumulated detections over all observations.

\section{ENVIRONMENTAL OBSERVATIONS USING DIGITAL BROADCASTS}

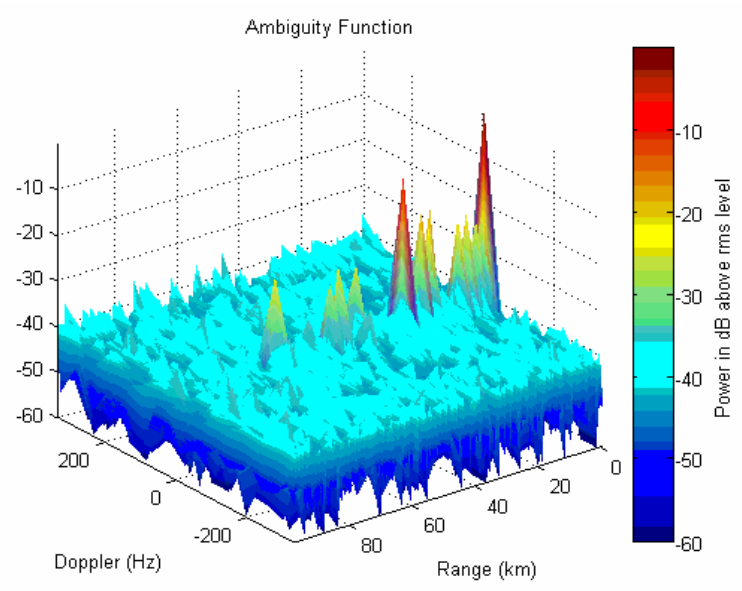

Figure 12 - Ambiguity function for vertical and horizontal dipoles.

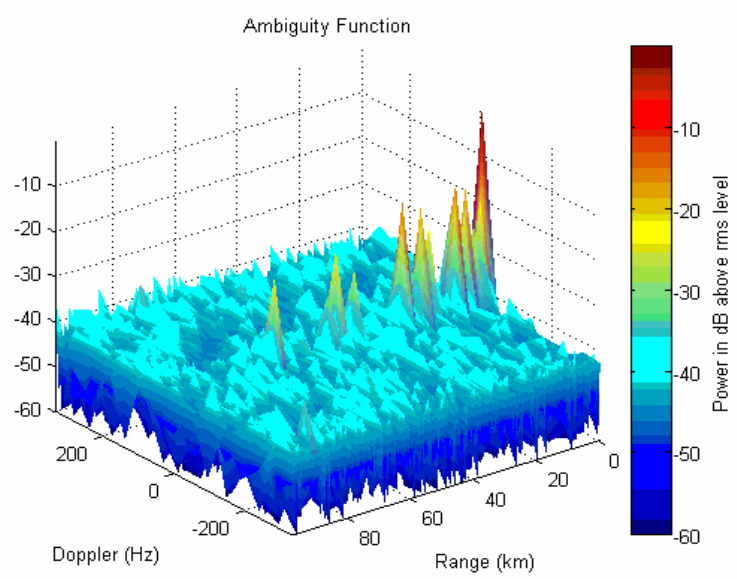

Figure 13 - Ambiguity functions two days later. 


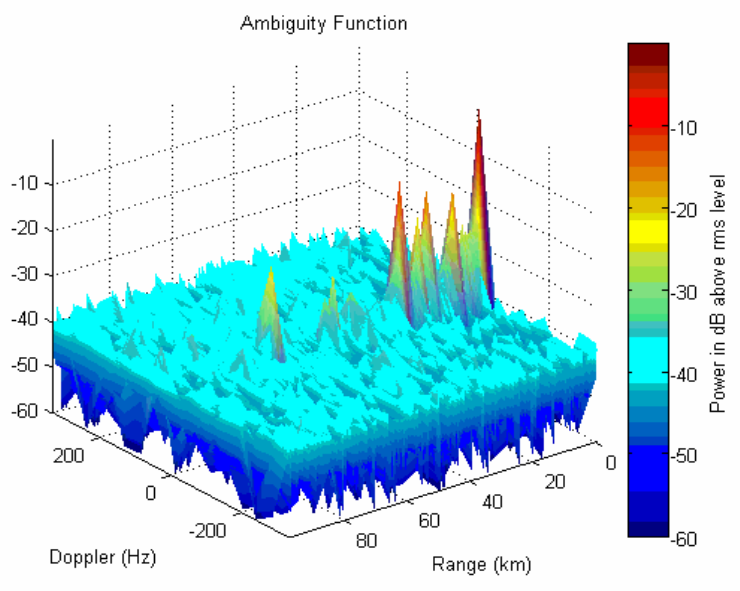

Figure 14 - Ambiguity function four days later.

One of the interesting features of DAB is that there exists an extensive network of transmitters across the UK and hence, at any given location, there will be signals from a multitude of DAB sources over a wide area (typically 5 or 6 in view at any one time). These signals will be affected by the propagation environment and, if this varies over time, the relative strengths and phases of these signals will also vary. Variations in temperature, pressure and humidity will cause variations in refractive index and this will in turn affect the propagation of the DAB signals. This variation will typically manifest itself as small fluctuations in phase. In extreme circumstances, however, the variation will cause ducting and result in very distant transmitters becoming visible. By placing a receiver in close proximity to one of the DAB transmitters, it is possible to make comparisons of distant transmissions with a signal that is relatively unaffected by the environment. Observations of the signals over time will provide information about the evolution of refractive index and hence about variations in temperature, pressure and humidity. Furthermore, the existence of a network of illuminators brings about the possibility of obtaining sufficient propagation data for inversion into a map of refractive index. Figs. 12 to 14 give some idea of the possibilities. They show the ambiguity function from DAB signals recorded on a vertical and horizontal dipoles close to a low power DAB transmitter at Bathampton near Bath (this provides the signal that is relatively unaffected by propagation). The cross products with this signal in the ambiguity function have suffered significant change over the time (two days have elapsed between the measurements represented by each figure), indicating that there is significant change in propagation conditions. Over the short term (minutes), significant fluctuations in amplitude have been noted. Consequently, there may be the possibility of monitoring atmospheric turbulence.

DAB transmissions also have the possibility of providing illuminations for passive radar studies of ionospheric structure through the scattering of VHF signals by the turbulence that exists in certain regions of the ionosphere (auroral and equatorial regions). Standard FM broadcasts have been successfully used for this purpose [10], but the more desirable properties of DAB transmissions could make it a useful alternative. Furthermore, the existence of a coherent system of illuminators could bring about the possibility of far more sophisticated imaging of the ionosphere.

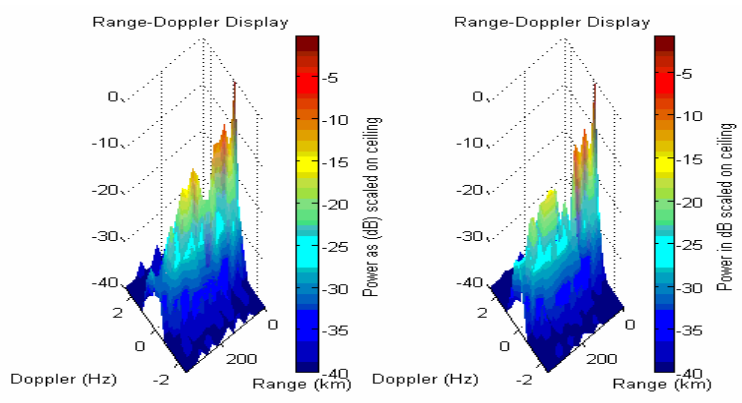

Figure 15 - Vertical and horizontal DRM signals at time 1.
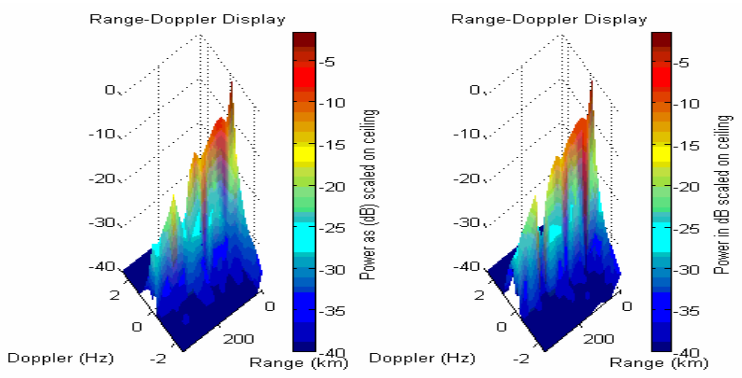

Figure 16 - Vertical and horizontal DRM signals at time 2.
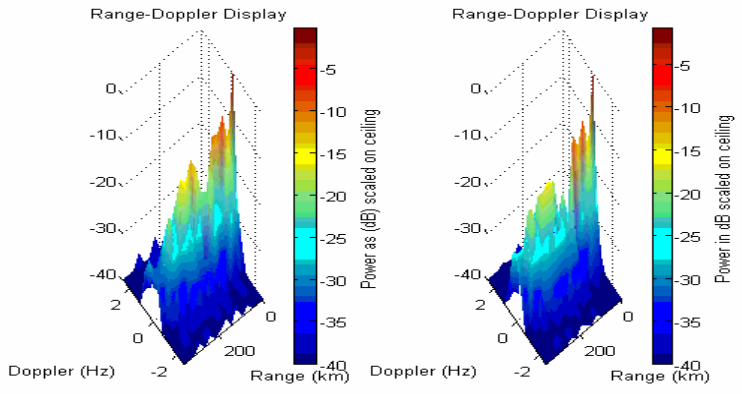

Figure 17 - Vertical and horizontal DRM signals at time 3.

DRM also has potential for the study of radio wave propagation through the ionosphere. The bandwidth of DRM is sufficiently wide to resolve the different propagation modes that occur in short wave propagation. This can be seen from fig. 15 which shows the ambiguity function for a DRM transmission received on both horizontal and vertical dipole antennas (the frequency was around $4 \mathrm{MHz}$ during early morning and the signal was from a transmitter at several thousand kilometers distance). Figs. 16 and 17 show observations a short time later (figures 15 to 17 spanning about half a minute) and from which it can be seen that there is a large variation in the amplitudes and polarization of the various modes. The ability to dissect a DRM signal into its constituent modes is an important tool in the study of propagation and the large number of transmitters that can be received at any one receiving site mean that quite extensive propagation studies can be accomplished with a single re- 
ceiver. In particular, the authors are currently using DRM observations to study the phenomenon of fading on shortwave transmissions.

\section{CONCLUSION}

This paper has described some aircraft observations using a passive bistatic radar that has been built by the University of Adelaide. The work has established that air traffic radar, based on DAB transmitters of opportunity, is feasible. In particular, the major problem of direct signal interference has been overcome using novel hardware and software. The next stage will be to develop the system so that it can also handle DVB signals and hence further increase the flexibility of the system. Further work still needs to be done on DSI removal and the issue of tracking needs to be addressed.

DRM also has potential for aircraft observations, but the range resolution is limited due to its very much lower bandwidth when compared to DAB. The radar test bed can also operate on DRM frequencies and such experiments are planned for the future. The number of DRM sites, however, is very limited and not particularly close to suitable air lanes. Typically, DRM has much higher power than DAB and the lower frequency can also work to its advantage. Consequently, it should be possible to detect aircraft at much greater ranges. Although there is the possibility of over the horizon radar using DRM, the available power is probably insufficient for sky wave radar unless an extremely large receiver array aperture is used. Surface wave radar, however, may be possible using low frequency coastal DRM transmitters.

A further possibility for passive radar that is based on digital broadcasts is that of monitoring the atmosphere. Radars based on FM broadcasts have already been used for this purpose and there is no reason why the radar test bed described in this paper could not also be used for such purposes. Future experiments of this nature are planned.

\section{REFERENCES}

[1] H.D.Griffiths, "From a different perspective: principles, practice and potential of bistatic radar", Proceedings of the IEEE Conference on Radar 2003, Adelaide, 2003.

[2] I.Baniak, I., G.Baker, A.M.Cunningham and L.Martin, "Silent Sentry passive surveillance", Aviation Week and Space Technology, 7 June 1999.

[3] H.D.Griffiths and C.J.Baker, "Measurement and analysis of ambiguity functions of passive radar transmissions", Proceedings of the IEEE Conference on Radar 2003, Adelaide, 2003.

[4] ] H.D.Griffiths and C.J.Baker, "Passive coherent location radar systems. Part 1: performance prediction”, IEE Proceedings in Radar, Sonar and Navigation, vol. 152, pp. 153 - 159, 2005.

[5] C.J.Baker, H.D.Griffiths and I.Papoutsis, "Passive coherent location radar systems. Part 2: waveform properties", IEE Proceedings in Radar, Sonar and Navigation, vol. 152, pp. 160 - 168, 2005.

[6] P.E.Howland, D.Maksimiuk and G.Reitsma, "FM radio based bistatic radar" IEE Proceedings in Radar, Sonar and Navigation, vol. 152, pp.107 - 115, 2005.

[7] R.Saini and M.Cherniakov, "DTV signal ambiguity function analysis for radar application", IEE Proceedings in Radar, Sonar and Navigation, vol. 152, pp. 133 - 142, 2005.

[8] D.Poullin, "Passive Detection using broadcasters (DAB, DVB) with CODFM modulation', IEE Pro. RSN, Vol.152, pp. 143-152, 2005.

[9] H.Yardley, "Bistatic Radar Based on DAB Illuminators: The Evolution of a Practical System", Proceedings of the IEEE Conference on Radar 2007, Boston, 2007.

[10] J.D.Sahr and M.Meyer, "Opportunities for passive VHF radar studies of plasma irregularities in the equatorial $\mathrm{E}$ and $\mathrm{F}$ regions", Journal of Atmospheric and Solar Terrestrial Physics, vol. 66, pp. 1675-1681 